\title{
New EU clinical trials regulation
}

\author{
Needs a few tweaks before implementation
}

\author{
Anna E Westra postdoctoral researcher in medical ethics ${ }^{1}$, Wendy Bos junior researcher ${ }^{1}$, Adam \\ F Cohen chief executive officer ${ }^{2}$
}

${ }^{1}$ Erasmus University Medical Centre, Department of Medical Ethics and Philosophy of Medicine, 3000 CA Rotterdam, Netherlands; ${ }^{2}$ Centre for Human Drug Research, Zernikedreef 10, 2333 CL, Leiden, Netherlands

On 2 April 2014, the European Parliament approved the new European Union Clinical Trials Regulation. ${ }^{1}$ This regulation will replace the Clinical Trials Directive, which has failed to achieve its goal of simplifying the scientific and ethical review of clinical trials in the EU. ${ }^{23}$ Unlike the directive, the regulation has binding legal force in all EU member states. Important innovations include a central database and a partly coordinated review system. Both of these steps could help simplify the review system and improve the quality of assessments.

However, when it drafted the regulation, quality improvement did not seem to be the European Commission's main concern. Despite serious criticisms and several adjustments, the approved document may still impair, rather than improve, the quality of the ethical review of trial protocols. ${ }^{4-9}$ This puts the protection of European research subjects at risk.

Before discussing our two main concerns, we need to explain the new review system. Currently, all member states assess the request for authorisation of a multinational clinical trial independently of one another. To simplify and speed up authorisation, the European Commission has decided that the risk-benefit assessment (and the preceding scientific assessment) should be performed in a coordinated manner. ${ }^{59}$ With this in mind, sponsors propose one member state to be the reporting one, and this member state makes the final decision on the risk-benefit assessment. The other member states are asked for their input, but within a very tight time frame. Their main task is to assess the ethical and local aspects, such as the informed consent material, the investigators' qualifications, and the suitability of the trial site, for their own territory. Thus, two types of assessment run in parallel: the coordinated risk-benefit assessment (by the reporting member state, binding on all member states), and the assessment of the ethical and local aspects mentioned above (by all member states acting individually).

We approve the idea of coordinating the assessment of multinational clinical trials. However, in the case of such centralised judgments, the quality of these judgments should be guaranteed. This is not the case.
We are mostly worried about the risk-benefit assessment being taken out of the ethical domain. The European Commission fails to acknowledge that this assessment is widely regarded as a crucial part of ethical review. ${ }^{10}$ As a result, the regulation does not require input from an ethics committee. ${ }^{9}$ This is worrying because the purpose of ethics committees is to focus on the protection of potential research subjects. This perspective is indispensable when the risks of harm and potential benefits of a clinical trial are being assessed. It is worth noting that studies may be ethically unacceptable, despite having a scientifically favourable harm-benefit balance, if the research question could be answered with fewer risks or burdens for the research subjects.

Furthermore, some studies have a favourable harm-benefit balance because of the expected benefits for society but are not expected to benefit the research subjects themselves. Such studies should be evaluated very carefully, particularly when they involve children or others who are considered unable to provide informed consent. ${ }^{10}$ The regulation provides guidelines for research in these groups, but applying these guidelines appropriately requires specific ethical expertise. ${ }^{9}$ Ideally, a multidisciplinary committee with wide ethical expertise should critically assess the risks and potential benefits and demand changes in the design when needed.

A complicating factor is that sponsors are free to choose the reporting member state. This might tempt sponsors to choose member states that are known for their less onerous assessments. Thus, when aiming for high quality review, the question of which body should perform the risk-benefit assessment cannot be left to the member states' own discretion.

Our second concern relates to the quality of the ethics committees. During the first public consultation round many respondents asked for quality standards and an accreditation system for these committees, ${ }^{4}$ but the European Commission has ducked this request - a truly missed opportunity. This is because even though ethical matters are regarded as a national affair, the new regulation would provide a great opportunity for improving the widely varying quality of the EU's ethics 
committees by setting clear quality standards. ${ }^{11}$ Leaving these committees just as diverse as before means that European citizens of different member states cannot rely on the same level of protection. Moreover, if ethics committees are also involved in the coordinated risk-benefit assessment, which we have just argued for, every opportunity to improve their quality should be taken. The judgment of the ethics committee of the reporting member state will then cover the protection of the research subjects in all member states.

We recognise that it will be difficult to make substantial changes to the new regulation now that it has been approved. However, it is wrong to rush through a system that is clearly inadequate. European research subjects deserve a clinical trials regulation that has a sound ethical basis. Therefore, we recommend that this new legislation is adjusted before coming into force.

In practical terms, we recommend that the ethics committee of the reporting member state should be assigned as the key figure in an integrated risk-benefit assessment system. In addition, this committee should be allowed enough time to take scientific advice from experts and to cooperate effectively with the ethics committees of the other member states. Lastly, a quality and accreditation system should be established for all ethics committees so that all trials are reviewed by competent committees.

Competing interests: We have read and understood BMJ policy on declaration of interests and declare the following interests: AFC is a member of the Medical Sciences Committee of Science Europe.
Provenance and peer review: Not commissioned; externally peer reviewed.

1 European Parliament. Clinical trials: clearer rules, better protection for patients. 2014. www.europarl.europa.eu/news/en/news-room/content/20140331IPR41186/html/Clinicaltrials-clearer-rules-better-protection-for-patients.

2 Directive 2001/20/EC of the European Parliament and of the Council of 4 April 2001 on the approximation of the laws, regulations and administrative provisions of the Member States relating to the implementation of good clinical practice in the conduct of clinical trials on medicinal products for human use. Official Journal of the European Communities 1.5.2001: L121/34-L121/44. www.eortc.be/services/doc/clinical-eu-directive-04-april-01. pdf.

3 European Commission. Assessment of the functioning of the "Clinical Trials Directive" 2001/20/EC. Public consultation paper. 2009. http://ec.europa.eu/health/files/clinicaltrials/ docs/2009_10_09_public-consultation-paper.pdf.

4 European Commission. Assessment of the functioning of the "Clinical Trials Directive" 2001/20/EC. Summary of responses to the public consultation paper. 2010. http://ec. europa.eu/health/files/clinicaltrials/2010_03_30_summary_responses.pdf.

5 European Commission. Revision of the "Clinical Trials Directive." Concept paper submitted for public consultation. Brussels: European Commission, 2011. http://ec.europa.eu/health/ files/clinicaltrials/concept_paper_02-2011.pdf.

6 European Commission. Revision of the "Clinical Trials Directive." Summary of the replies to the public consultation on the "Concept paper." 2011. http://ec.europa.eu/health/files/ clinicaltrials/ctresp_2011-06/ct_summary.pdf.

7 Tuffs A. Germany opposes EU plans for regulating clinical trials owing to lack of ethical standards. BMJ 2012;345:e6640.

8 Hasford J. New European Union regulation of clinical trials puts trial participants at risk. BMJ 2013;346:f665.

9 Council of the European Union. Proposal for a regulation of the European Parliament and of the council on clinical trials on medicinal products for human use, and repealing Directive 2001/20/EC. 2013. http://ec.europa.eu/health/files/clinicaltrials/2012_07/proposal/2012 07_proposal_en.pdf.

10 Emanuel EJ, Wendler D, Grady C. What makes clinical research ethical? JAMA 2000;283:2701-11.

11 Kenter MJH, Cohen A. Re-engineering the European Union Clinical Trials Directive. Lancet 2012;379:1765-7.

Cite this as: BMJ 2014;348:g3710

๑ BMJ Publishing Group Ltd 2014 